\title{
Glycogenic Hepatopathy With Persistent Hepatomegaly in a Patient With Uncontrolled Type 1 Diabetes
}

\author{
Ravi Kant ${ }^{\mathrm{a}, \mathrm{f}}$, Hillary B. Loper ${ }^{\mathrm{b}}$, Vipin Verma ${ }^{\mathrm{c}}$, Rana Malek ${ }^{\mathrm{d}}$, Cinthia B. Drachenberg ${ }^{\mathrm{e}}$, Kashif M. Munir ${ }^{\mathrm{d}}$
}

\begin{abstract}
Even though glycogen overloading of the liver was first described as a component of Mauriac syndrome in 1930, glycogenic hepatopathy (GH) is still an under-recognized complication of type 1 diabetes mellitus (T1DM). GH diagnosis is challenging as it is clinically difficult to distinguish GH from steatohepatitis or glycogen storage disease, and liver biopsy is essential for confirmatory diagnosis. GH is usually a transient condition with unknown long-term complications. We present a case of GH with persistent marked hepatomegaly over 5 years despite normalization of liver enzymes in a patient with uncontrolled T1DM.
\end{abstract}

Keywords: Type 1 diabetes mellitus; Glycogenic hepatopathy; Hepatomegaly; Transaminitis

\section{Introduction}

Even though glycogen overloading of the liver was first described as a component of Mauriac syndrome in 1930 [1], glycogenic hepatopathy $(\mathrm{GH})$ is still an under-recognized complication of type 1 diabetes mellitus (T1DM). Pathogenesis

\footnotetext{
Manuscript accepted for publication April 09, 2015

aDepartment of Family Medicine (Anderson), Medical University of South Carolina AHEC and Division of Endocrinology, Edward Via College of Osteopathic Medicine (VCOM), 100 Healthy Way, Suite 1210, Anderson, SC 29621, USA

bLifebridge Health, 750 Main Street, Suite 310, Reisterstown, MD 21136, USA

'Department of Internal Medicine, Georgetown University Hospital/Washington Hospital Center, 110 Irving St NW, Washington, DC 20010, USA

${ }^{\mathrm{d} D i v i s i o n}$ of Endocrinology, Diabetes and Nutrition, University of Maryland School of Medicine, 827 Linden Avenue, Baltimore, MD 21201, USA

eDepartment of Pathology, University of Maryland School of Medicine, 22 South Greene St., Baltimore, MD 21201, USA

${ }^{f}$ Corresponding Author: Ravi Kant, Department of Family Medicine (Anderson), Medical University of South Carolina AHEC and Division of Endocrinology, Edward Via College of Osteopathic Medicine (VCOM), 100 Healthy Way, Suite 1210, Anderson, SC 29621, USA. Email: rkant82@hotmail.com
}

doi: http://dx.doi.org/10.14740/jem276w of GH is incompletely understood but clinical presentation is fairly well defined. GH typically presents with abdominal pain, marked hepatomegaly and transaminitis in patients with poorly controlled T1DM. Although several recent cases report resolution of these clinical abnormalities with improved glycemic control, knowledge of long-term outcomes of these patients is still insufficient [2-7]. We present a case of GH with persistent marked hepatomegaly over 5 years despite normalization of liver enzymes in a patient with uncontrolled T1DM.

\section{Case Report}

A 15-year-old male with T1DM for 5 years presented with abdominal pain and diabetic ketoacidosis (DKA). Physical examination revealed marked hepatomegaly. Laboratory analysis showed HbA1c 11.4\%, AST 3,965 (8 - 43 U/L), ALT 1,275 (7 - $45 \mathrm{U} / \mathrm{L})$, ALP 196 (41 - $108 \mathrm{U} / \mathrm{L})$, total bilirubin 1.5 (0.1 - 1.0 $\mathrm{mg} / \mathrm{dL}$ ), albumin $2.8 \mathrm{~g} / \mathrm{dL}$ and a normal coagulation profile. $\mathrm{Ab}-$ dominal ultrasound (US) demonstrated hepatomegaly $(>21 \mathrm{~cm})$ with diffusely increased liver echogenicity consistent with fatty infiltration. Abdominal computed tomography (CT) confirmed hepatomegaly $(23 \mathrm{~cm})$. A comprehensive laboratory evaluation looking for the underlying etiology was unremarkable, excluding autoimmune hepatitis, Wilson's disease, hemochromatosis, celiac disease and viral infections (hepatitis A, B, C, CMV and EBV). Liver biopsy results are shown in Figure 1. Patient was diagnosed with GH and initiated on basal-bolus insulin therapy. The patient had three episodes of DKA over the ensuing 5 years. Transaminitis resolved 5 months after the initial presentation, but abdominal US and CT 1 year and 5 years later, respectively, demonstrated persistent but stable hepatomegaly.

\section{Discussion}

Our case demonstrated marked liver dysfunction and hepatomegaly during an episode of DKA. Interestingly, liver dysfunction, even though severe, resolved spontaneously via glycemic control without any specific treatment. This transient elevation of liver enzymes in an uncontrolled T1DM patient is consistent with a diagnosis of $\mathrm{GH}$, as reported previously by several investigators [2-7]. GH is usually a transient condition with unknown long-term complications. Our case is unique given 

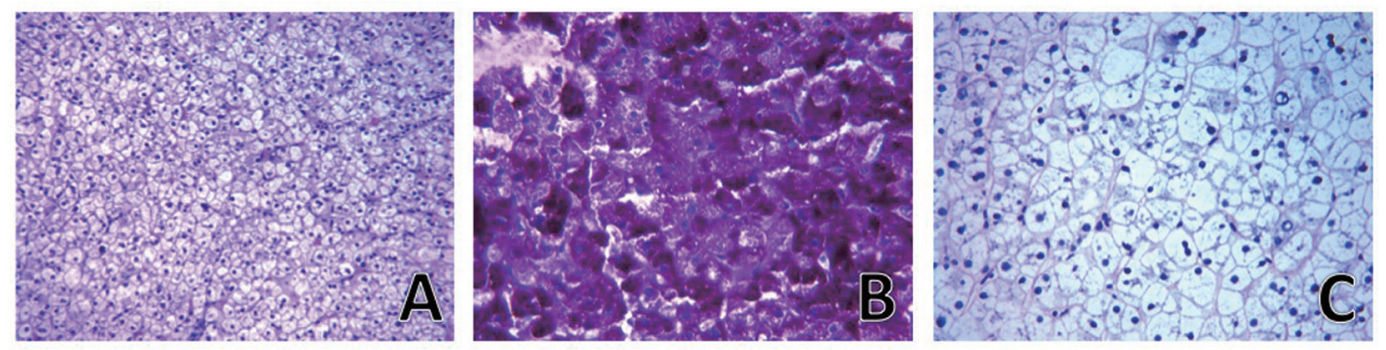

Figure 1. Liver biopsy. Hematoxylin and eosin stain (A) showing marked cleaning of the hepatocytes cytoplasm but intact liver architecture with no significant inflammation or fibrosis and periodic acid-Schiff stain (B) demonstrating abundant cytoplasmic glycogen deposits, which disappeared after digestion with diastase (C) consistent with diagnosis of glycogenic hepatopathy.

persistent marked hepatomegaly over 5 years despite normalization of liver enzymes in a patient with GH.

Insulin treatment in patients with uncontrolled T1 DM has been shown to influence hepatocytes, resulting in transient transaminitis. In a retrospective chart review, Takaike et al [8] detected transient elevation of liver enzymes during the first month after insulin initiation in $29.1 \%$ and $60.4 \%$ of patients with acute-onset T1DM and fulminant T1DM, respectively. This observation, however, did not affect the treatment plan, probably due to its transient nature. Additionally, non-alcoholic fatty liver disease (NAFLD) was diagnosed in $65 \%$ of patients with T1DM demonstrating transaminitis and $20 \%$ of patients with new-onset T1DM. Since NAFLD is pathologically linked to a state of insulin resistance and metabolic syndrome, such high prevalence of this disorder in T1DM was surprising. Because liver biopsy is not routinely performed and diagnosis of NAFLD is made clinically based on US and CT findings, it is possible that many cases of $\mathrm{GH}$ might be inappropriately diagnosed as NAFLD. Our case was also initially misdiagnosed as NAFLD based on US characteristics.

While most liver dysfunction associated with insulin treatment in uncontrolled T1DM patients is mild and does not alter management, evidence has accumulated over the last decade that transaminitis can be severe, leading to unnecessary investigation and invasive procedures [6, 7]. Our patient underwent extensive evaluation including laboratory and imaging tests looking for underlying etiologies prior to getting a liver biopsy, which definitively diagnosed GH. Since GH is a transient clinical condition that resolves with improved glycemic control, it is critical for clinicians to be aware of this underrecognized complication of uncontrolled T1DM. It is clinically challenging to distinguish $\mathrm{GH}$ from steatohepatitis or glycogen storage disease, and liver biopsy is essential for confirmatory diagnosis. Intact architecture with no significant fibrosis and marked hepatocellular glycogen accumulation are histological hallmarks of $\mathrm{GH}[3]$.

Various imaging studies have been suggested to differentiate glycogen deposition from fat deposition in liver. US is not a useful modality in diagnosis of $\mathrm{GH}$, as it cannot differentiate fat from glycogen. CT density, however, usually increases with accumulation of glycogen content and decreases in patients with fatty liver [7, 9]. For this reason, some authors have argued that increased liver density could be a clue to diagnosing GH. Sensitivity of CT, however, is limited in detecting GH. Murata et al [6] reported a case of GH with diffusely decreased
CT density in the liver, which was thought to be due to shock liver. Additionally, authors have claimed that gradient-dualecho MRI sequence could be a clue to diagnose GH due to its ability to distinguish a fat deposition from an edematous condition, such as acute liver failure [6]. Even though liver biopsy is necessary to diagnose GH with certainty, a combination of non-invasive studies in classical clinical scenario may point to a diagnosis of $\mathrm{GH}$.

Hepatomegaly in GH typically resolves with improved diabetes management. However, to our knowledge this is the first published case to demonstrate persistent marked hepatomegaly over 5 years despite normalization of liver enzymes in a patient with GH. This is probably due to the patient's nonadherence to his basal-bolus insulin regimen, leading to persistently uncontrolled T1DM with multiple episodes of DKA. The absence of clinically significant progression of liver disease over 5 years remains striking, although development of fibrotic changes in his liver cannot be completely excluded, as follow-up liver biopsy was not performed. This report illustrates the fact that even marked transaminitis ( $>90$ times normal) associated with GH is usually transient, but hepatomegaly can be persistent. Long-term consequence of this observation is unknown. Increased awareness among clinicians is required to diagnose this potentially reversible under-recognized T1DM complication.

\section{Disclosure}

The authors have nothing to disclose.

\section{References}

1. Mauriac P. Gros ventre, hepatomegalie, troubles de las croissance chez les enfants diabetiques traits depuis plusieurs annes par l'insuline. Gax Hebd Med Bordeaux. 1930;26:402-410.

2. Brouwers MC, Ham JC, Wisse E, Misra S, Landewe S, Rosenthal M, Patel D, et al. Elevated lactate levels in patients with poorly regulated type 1 diabetes and glycogenic hepatopathy: a new feature of Mauriac syndrome. Diabetes Care. 2015;38(2):e11-12.

3. Torbenson M, Chen YY, Brunt E, Cummings OW, Gottfried M, Jakate S, Liu YC, et al. Glycogenic hepatopa- 
thy: an underrecognized hepatic complication of diabetes mellitus. Am J Surg Pathol. 2006;30(4):508-513.

4. Rogal SS, Ukomadu C, Levy BD, Loscalzo J. Clinical problem-solving. A sweet source of abdominal pain. $\mathrm{N}$ Engl J Med. 2011;364(18):1762-1767.

5. Imtiaz KE, Healy C, Sharif S, Drake I, Awan F, Riley J, Karlson F. Glycogenic hepatopathy in type 1 diabetes: an underrecognized condition. Diabetes Care. 2013;36(1):e6-7.

6. Murata F, Horie I, Ando T, Isomoto E, Hayashi H, Akazawa S, Ueki I, et al. A case of glycogenic hepatopathy developed in a patient with new-onset fulminant type 1 diabetes: the role of image modalities in diagnosing hepatic glycogen deposition including gradient-dual-echo
MRI. Endocr J. 2012;59(8):669-676.

7. Sweetser S, Kraichely RE. The bright liver of glycogenic hepatopathy. Hepatology. 2010;51(2):711-712.

8. Takaike H, Uchigata Y, Iwamoto Y, Imagawa A, Iwahashi H, Kanatsuka A, Kawasaki E, et al. Nationwide survey to compare the prevalence of transient elevation of liver transaminase during treatment of diabetic ketosis or ketoacidosis in new-onset acute and fulminant type 1 diabetes mellitus. Ann Med. 2008;40(5):395-400.

9. Tsujimoto T, Takano M, Nishiofuku M, Yoshiji H, Matsumura Y, Kuriyama S, Uemura M, et al. Rapid onset of glycogen storage hepatomegaly in a type-2 diabetic patient after a massive dose of long-acting insulin and large doses of glucose. Intern Med. 2006;45(7):469-473. 